\title{
关于准晶体结构的一些数学回答
}

1984 年 11 月, D. Shechtman 等人在急冷的 镇-铝合金中拍摄到第一张准晶体的电子衍射图. 这个发现冲破了一百多年来建立起来的经典晶体学 的现有理论基础。在随后得到的高分辨电子显微图 中,呈圆环状分布的亮点在直线方向或相间,或重叠, 而结点分布服 从 Fibonacci 排列 (cf. K. Hiraga et al., Science Reports Research Institutes TÔHOKU University Ser. A. V. $32(1984 / 85) 309)$. 本文提出 的“M-L 排队规则”和有关定理，较圆满地描述了 这一现象. 我们期望它们将成为理想准晶体结构数 学描述的某些基础.

M-L 排队规则 设 L 为平面上一条直线, 取定一点记为 0 , 和正方向. 对 L 上的任一点 $a(>$ 0 ), 若以 0 为心， $a$ 为半径的圆在 L 正向上按下述 规则生成一簇圆,

(i) 以 $\lambda a$ 为圆心 $(2<\lambda<\sqrt{3}+1)$, $a$ 作半径 作圆, 该圆与 $\mathrm{L}$ 的两个交点和圆心再分别放大 $\lambda$ 倍 后, 并以 $a$ 为半径旋转且按规则 (ii), 形成新的圆 或重叠圆,如此进行至无穷.

(ii) 若按上述方法, 形成的两个相邻的圆不重 叠, 则两圆之间的距离为 $b$; 若两个相邻圆重叠, 则
认定两圆重叠的部分为先生成的圆所遮盖, 并要求 后生成圆的圆心与先生成的圆之距离为 $b$.

则上述规则我们称为 $\mathrm{M}-\mathrm{L}$ 排队规则.

定义 若 $a 、 b$ 为两个符号, 记 $\mathscr{F}_{0}=b, \mathscr{F}_{1}=a$; $\mathscr{F}_{n}=\mathscr{F}_{n-1} \mathscr{F}_{n-2}(n \geq 2), \mathscr{F}_{n}$ 表示 $\mathscr{F}_{n-1}$ 与 $\mathscr{F}_{n-2}$ 先后衔接的符号排列, 称为 Fibonacci 排列. 若记 $\bar{F}_{0}=\bar{F}_{1}=1, \bar{F}_{n}=\bar{F}_{n-1}+\bar{F}_{n-2}, n \geq 2$. 则称 $\bar{F}_{n}$ 为 Fibonacci 数.

定理 1 按 $\mathrm{M}-\mathrm{L}$ 排队规则生成的一簇圆周(带 圆心）与过其圆簇心直线 L 的交点之间的距离变化 服从 Fibonacci 排列. 且必有

$$
\lambda=\left(\frac{1+\sqrt{5}}{2}\right)^{2} ; a: b=\frac{1+\sqrt{5}}{2} .
$$

定理 2 对于任意 $\mathscr{F}_{n}$ 中的第 $\mathrm{N}$ 号元素 $\mathrm{x}$, 可由 $\mathrm{N}=\sum_{i=1}^{k} \bar{F}_{n_{i}}$ 来判定, 其中 $n_{i} \geq n_{i+1}+2, i=1$, $2, \cdots, k-1, k \leqslant \frac{n_{1}+2}{2}$. 当 $n_{k}$ 为奇数时 $\mathrm{x}$ 是 $a$; $n_{k}$ 为偶数时 $\mathrm{x}$ 是 $b$.

闵乐泉 李宗元 (北京钢铁学院数力系)

\section{Frankia 纯培养的无细胞抽提液固氮}

自从 1960 年 Carnahan 等人证明巴区梭菌粗 抽提液的固氮酶活性以来,迄今已从大约 20 种不同 微生物中获得了含固氮酶活性无细胞制剂, 并对其 中一些固氮酶的性质作了详细研究. 但对非豆科树 木形成固氮根㾕的 Frankia 内生菌纯培养尚末见报 道.

为了研究 Frankia 内生菌固氮酶的特性, 我们 首先确定了它的扩大培养和合成固氮酶的条件, 并 在获得较大量菌体的基础上, 研究了制备具有固氮 活性的 Frankia 纯培养的无细胞抽提液的方法. 本文是这一方法的简要介绍.

1.制备步啋：离心收集具活泼乙炔还原活性的
科学通报
Frankia 内生菌培养物, 并用 $25 \mathrm{mmol} / 1 \mathrm{Tris}-\mathrm{HCl}$ 缓冲 液 ( $\mathrm{pH7}$.4) 洗一次. 然后, 在氮气流下, 将洗过的 菌体悬浮在适量的含 $0.3 \mathrm{mg} \mathrm{Na} \mathrm{S}_{2} \mathrm{O}_{4} / \mathrm{ml} 、 0.2 \mathrm{mg}$ 二硫 苏糖醇 (DTT) $/ \mathrm{ml}$ 的 $25 \mathrm{mmol} / 1$ Tris-HCl 缓冲液 (pH7.4) 中, 并用 $150 \mathrm{~W}$ 的 MSE 超声波发生器于 满功率 $F$ 超声破碎三次, 每次 $3 \mathrm{~min}$, 冷却间歇 $1 \mathrm{~min}$ 钟. 破碎液在罣气下、 $4^{\circ} \mathrm{C} 、 15000 \mathrm{~g}$ 离心 $30 \mathrm{~min}$, 除 去细胞碎片. 对所得上清液 (即无细胞抽提液)进行 固氮酶乙炔还原活性分析.

2.固氮酶乙炔还原活性分析：测定在容积 $7 \mathrm{ml}$

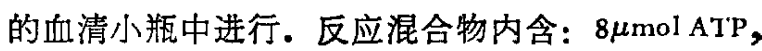
$15 \mu \mathrm{mol} \mathrm{MgCl}_{2}, 51 \mu \mathrm{mol} \mathrm{CP}$ (磷酸肌酸), $1.5 \mathrm{mg} \mathrm{CK}$ 